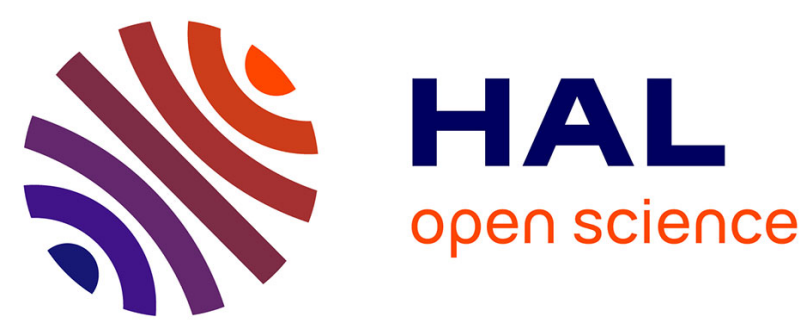

\title{
Graph Fourier Transform of fMRI temporal signals based on an averaged structural connectome for the classification of neuroimaging
}

Abdelbasset Brahim, Nicolas Farrugia

\section{> To cite this version:}

Abdelbasset Brahim, Nicolas Farrugia. Graph Fourier Transform of fMRI temporal signals based on an averaged structural connectome for the classification of neuroimaging. Artificial Intelligence in Medicine, 2020, 106, pp.101870. 10.1016/j.artmed.2020.101870 . hal-02302538v2

HAL Id: hal-02302538

https://hal-imt.archives-ouvertes.fr/hal-02302538v2

Submitted on 4 Mar 2020

HAL is a multi-disciplinary open access archive for the deposit and dissemination of scientific research documents, whether they are published or not. The documents may come from teaching and research institutions in France or abroad, or from public or private research centers.
L'archive ouverte pluridisciplinaire HAL, est destinée au dépôt et à la diffusion de documents scientifiques de niveau recherche, publiés ou non, émanant des établissements d'enseignement et de recherche français ou étrangers, des laboratoires publics ou privés. 


\title{
Graph Fourier Transform of fMRI temporal signals based on an averaged structural connectome for the classification of neuroimaging
}

\author{
Abdelbasset Brahim, Nicolas Farrugia \\ IMT Atlantique - Lab STICC, Department of Elecronics, Brest, France
}

\begin{abstract}
Graph signal processing (GSP) is a framework that enables the generalization of signal processing to multivariate signals described on graphs. In this paper, we present an approach based on Graph Fourier Transform (GFT) and machine learning for the analysis of resting-state functional magnetic resonance imaging (rs-fMRI). For each subject, we use rs-fMRI time series to compute several descriptive statistics in regions of interest (ROI). Next, these measures are considered as signals on an averaged structural graph built using tractography of the white matter of the brain, defined using the same ROI. GFT of these signals is computed using the structural graph as a support, and the obtained feature vectors are subsequently benchmarked in a supervised learning setting. Further analysis suggests that GFT using structural connectivity as a graph and the standard deviation of fMRI time series as signals leads to more accurate supervised classification using a world-wide multi-site database known as ABIDE (Autism Brain Imaging Data Exchange) when compared to several other statistical metrics. Moreover, the proposed approach outperforms several approaches, based on using functional connectomes or complex functional network measures as features for classification.
\end{abstract}

Keywords: Graph Signal Processing, Machine Learning, Resting-State Analysis, Neuroimaging, Classification 


\section{Introduction}

Functional magnetic resonance imaging (fMRI) is a noninvasive and safe imaging technique for measuring and mapping brain activity, and is commonly used in the field of cognitive neurosciences. However, the analysis of fMRI data 5 is a major challenge due to a high sensitivity to noise, a large number of dimensions for few observations per subject, or different acquisition protocols [1]. Recently, there has been an increasing interest in the application of multivariate analysis and machine learning to understand complex properties of brain networks and to assist diagnosis in brain imaging data [2, 3]. However, few analytical approaches take into account both the multivariate aspect and the connectivity features of the brain, such as a structural graph estimated using white matter tractography, or a functional connectivity graph computed using temporal covariation between neural activity time series.

As a potential answer to these challenges, Graph Signal Processing (GSP), which

15 is an emerging sub-field of signal processing, takes into account the underlying graphical structure of multivariate data. Moreover, it aims to generalize classical signal processing techniques, such as filtering, convolution, or translation to irregular graph domains [4. According to spectral graph theory [5], a Fourier transform can be defined on graphs from the eigendecomposition of the graph's Laplacian operator. Thus, GSP can be used to provide a spectral representation of signals defined on a graph, through the so-called Graph Fourier Transform operator (GFT). Therefore, GSP appears as an ideal framework to analyze fMRI data, as it makes it possible to consider brain activity defined on a brain connectivity graph [6].

25 On the other hand, several statistical features, such as the mean and the standard deviation (SD) of multivariate signals, have previously been used to compute a vector with discriminatory (spatial) features for disease classification [7]. Thus, in this work, we evaluate a GSP-based approach for the analysis and classification of neuroimaging data. We introduce a method based on resting so state functional magnetic resonance imaging (rs-fMRI), consisting in measuring 
the spontaneous brain activity of subjects at rest. Rs-fMRI is a very popular method, due to the simplicity of its protocol, the wide availability of data and analysis methods, and the large body of evidence regarding its functional and clinical relevance [8, [9. In our study, we assess whether the combination of

35 an average structural graph with several statistical metrics on rs-fMRI signals (such as SD) can extract meaningful features for the supervised classification of patients with autism spectrum disorder (ASD).

\subsection{Related work}

Several approaches to the classification of ASD patients using rs-fMRI have been proposed in the literature $3,10,16$. Most approaches have attempted to identify functional connectivity patterns that can discriminate patients from typical controls (TC) subjects using rs-fMRI. In [3], the authors obtained pairwise functional connectivity measurements from a lattice of 7266 regions of interest covering the gray matter for each subject. Then a leave-one-out classifier was evaluated on these connections, which were grouped into multiple bins. An accuracy rate of $60 \%$ was obtained for whole brain classification using the Autism Brain Imaging Data Exchange (ABIDE) datasets. In [10, the authors presented a mathematical framework based on Riemannian geometry and kernel methods that can be applied to connectivity matrices for the classification of ASD. Their approach achieved an accuracy value of $60.76 \%$. However, it was validated on a small dataset of only 79 subjects: $37 \mathrm{TC}$ and 42 pathological subjects. The authors in [11 developed a diagnostic approach, in which the correlation matrices computed from rs-fMRI time-series data were considered as features and entered into a probabilistic neural network (PNN) classifier to separate ASD from TC. The correlation matrices of 640 subjects were classified as ASD or TC with approximately $90 \%$ accuracy using the PNN algorithm. However, only subjects under 20 years of age were included in their study. In [12, the authors proposed a diagnostic framework for ASD, which is based on the computation of Pearson correlation-based functional connectivity network of each cluster. The 
clusters were obtained by the decomposition of rs-fMRI time series into distinct clusters with similar spatial distribution of neural activity. Their results achieved an accuracy rate of $71 \%$ and their framework was validated on several selected subjects from a subset of the ABIDE database, i.e. the New York University (NYU) Langone Medical Center. This dataset contains more than 170 subjects, but the authors included only 92 selected subjects in their study. The authors in 13 investigated several pipelines that extract the most predictive biomarkers from the data by building participant-specific connectomes from functionally-defined brain areas. These connectomes were then compared across participants to learn patterns of connectivity that differentiate TC from ASD patients. The best pipeline lead to $67 \%$ prediction accuracy on the full ABIDE database. In [14, the authors investigated patterns of functional connectivity that objectively identify ASD participants from rs-fMRI data using deep learning algorithms. Their results surpassed existing solutions by achieving $70 \%$ of accuracy in the identification of ASD versus controls in the ABIDE dataset. In [15], a novel metric learning method to evaluate the distance between functional connectivity graphs was proposed. This novel approach leverages the power of convolutional neural networks, while exploiting concepts from spectral graph theory to allow these operations on irregular graphs [15. The authors applied 80 the proposed model to functional brain connectivity graphs from the ABIDE database. Their experimental results show that their method can learn a graph similarity metric tailored for a clinical application, improving the performance of a simple $k$-nn classifier by $11.9 \%$ compared to a traditional distance metric, and a classification score of $62.9 \%$ was obtained using data from all the sites.

${ }_{85}$ The authors in [16] introduced a new biomarker extraction pipeline for ASD that relies on the use of graph-theoretical metrics of fMRI-based functional connectivity and machine learning algorithms. Their results suggest that measures of centrality provide the highest contribution to the classification power of a model for the $>30$ years age group, achieving an accuracy, sensitivity, and specificity

90 of 95,97 , and $95 \%$, respectively. However, their model is age-dependent and in this age range, there are only 51 subjects. Besides, the ABIDE dataset contains 
more than eight hundred subjects with a wide age range $(5-65)$.

\subsection{Contributions}

In this paper, we propose a novel multimodal analysis approach for brain imaging data, as shown in Figure 1 . This method of analysis combines GFT on a structural graph, and several statistical metrics of rs-fMRI time series defined on the same brain regions as the structural graph, hence using two different imaging modalities: rs-fMRI and diffusion-weighted imaging (DWI). The proposed approach comprises four stages. Firstly, the Glasser atlas is used for brain parcellation [17] to extract the rs-fMRI time series of each subject. These time series are summarized by several statistical metrics, such as the temporal average or SD. Secondly, the resulting statistical measures are projected onto an averaged structural graph of TC subjects from the Human Connectome Project (HCP) dataset using GFT. Then, in order to select the most informative features for classification, a univariate feature selection is performed using an analysis of variance (ANOVA). Finally, in order to keep the research finding more objective, two different classifiers are used to test the effectiveness of the proposed analysis method, namely a support vector machine (SVM) with linear kernel and a logistic regression classifier.

The proposed approach provides a different insight from previous methods [3, 13 16, namely:

- The proposed method does not exploit functional connectivity matrices directly from rs-fMRI, but rather relies on descriptive statistics of time series, combined with spatial and anatomical information using mean structural connectivity of several TC subjects and GFT;

- Our approach restores informative features related to neuro-psychiatric disease, such as ASD, as exemplified by statistically robust gains in classification metrics when compared to other feature extraction methods, including functional connectivity and graph theoretical metrics; 
- Taking into account the computational load, the proposed approach is less demanding when compared to functional connectomes-based approaches for the analysis of rs-fMRI brain data, as it is based only on the computation of statistical metrics;

\section{Materials and Methods}

\subsection{Database}

The data used in this study were collected from the Autism Brain Imag-

- The proposed approach decreases the amount of data needed to store patient imaging data history. Thus, each subject can be defined by several resulting features after their transformation using GFT defined on the same brain regions. For instance, the fMRI file for one time point for each patient is dependent on the number of volumes and the number of voxels or an intermediate method to store the square of the number of regions for the connectivity matrix. This file can be resumed only to the number of regions using the proposed approach;

- Our method performs efficient dimensionality reduction, by using a statistical criterion to select the most predictive features.

- We extend our previous results on the biggest subset of ABIDE, i.e. NYU Langone Medical Center [18, showing that the proposed analysis framework performs well in the discrimination task between ASD and TC subjects, and outperforms most prior work on this subset.

- Finally, the proposed method is validated on a large world-wide multi-site database (ABIDE) in which different methods of imaging acquisition were used [19].

Data Exchange (ABIDE) datasets 1 . ABIDE I consists of 1112 subjects

${ }^{1}$ See http://fcon_1000.projects.nitrc.org/indi/abide/abide_I.html for specific information. 


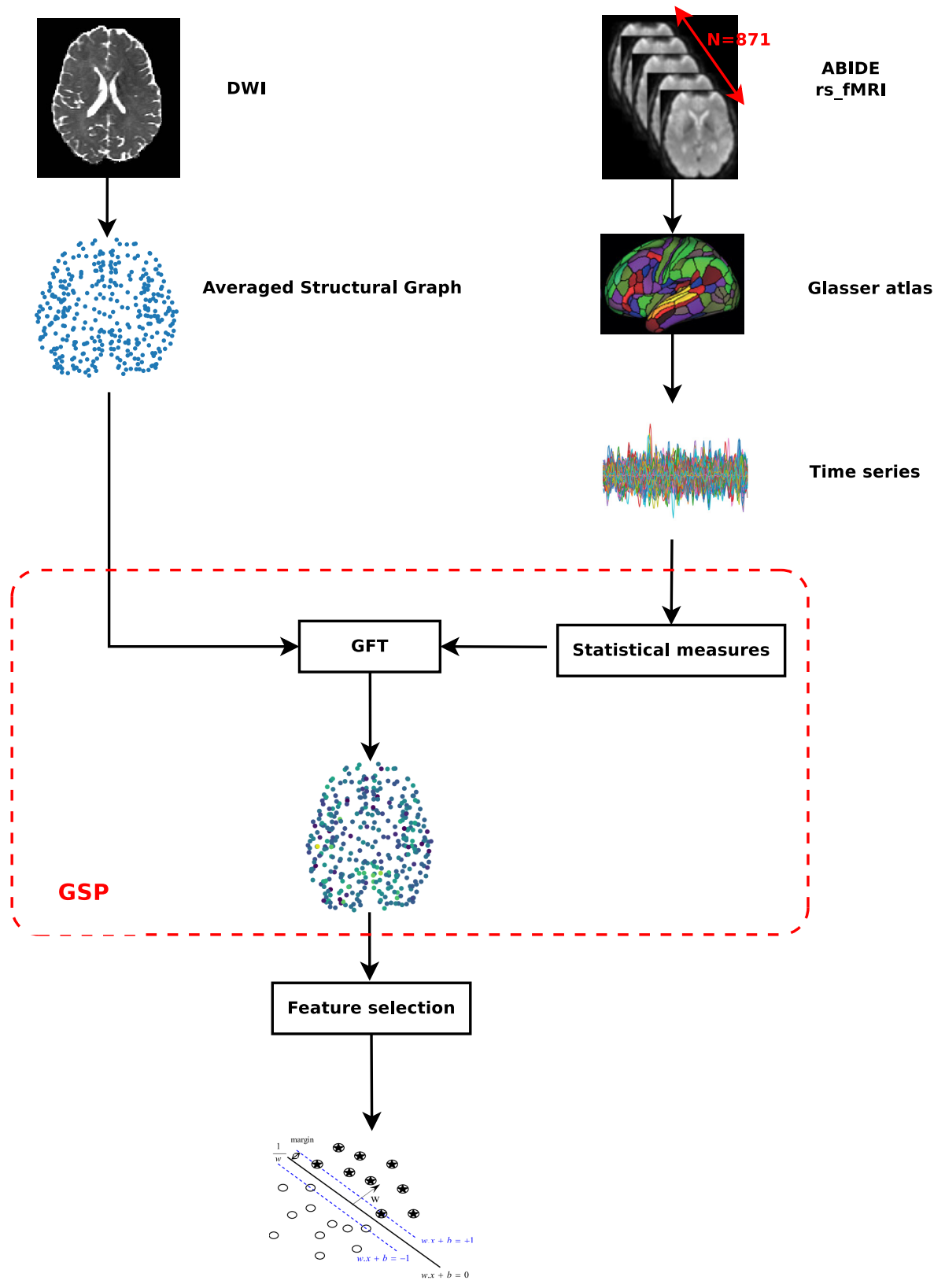

Figure 1: Diagram of the proposed approach. 
comprising 539 ASD patients and 573 TC subjects [19]. For easy replication, this database has previously been preprocessed by the Configurable Pipeline for the Analysis of Connectomes (C-PAC) 20. C-PAC uses several preprocessing techniques, such as skull striping, slice timing correction, motion correction, global mean intensity normalization, nuisance signal regression, band-pass filtering $(0.01-0.1 \mathrm{~Hz})$ and registration of fMRI images to standard anatomical MNI space. The selection of the data is based on the results of quality visual inspection by three experienced clinicians who checked for largely incomplete brain coverage, high movement peaks, ghosting and other scanner artifacts. This yielded 871 subjects out of the initial 1112, consisting of 403 individuals suffering from ASD and 468 TC. Due to the use of different acquisition protocols, the data is very different from one international site to another.

\subsection{Regions of interest and Time-series extraction}

The proposed approach is based, firstly, on regional time series extraction from brain parcellations. We used the Glasser parcellation [21], generated using multimodal data from the Human Connectome Project (HCP), totaling 360 regions. Thus, the time series of rs-fMRI brain imaging data were extracted according to 360 regions of interest (ROI) for each subject. Importantly, the same ROIs are defined in the structural graph that was used to establish GFT.

2.3. Graph Signal Processing and Graph Fourier Transform on Structural Graph

In this work, we are interested in the analysis of rs-fMRI signals on an averaged structural graph. Let us first define an undirected, connected, weighted and symmetric graph $\mathcal{G}=\{\mathcal{V}, \mathcal{E}, \mathbf{W}\}$. The graph is characterized by a finite set of vertices $\mathcal{V}$ indexed from 1 to $\mathrm{N}$ :

$$
\mathcal{V}=\left\{v_{1}, \ldots, v_{N}\right\}
$$

as well as a set of edges $\mathcal{E}$ in $\mathcal{V} \times \mathcal{V}$, and a weighted adjacency matrix $\mathbf{W}$, such that $\mathbf{W}_{i j} \in \mathbb{R}^{+}$denotes the weight of the edge $\left(v_{i}, v_{j}\right)$.

The combinatorial graph Laplacian of a graph is defined by [6, 22]:

$$
\mathbf{L}=\mathbf{D}-\mathbf{W}
$$


where $\mathbf{D}$ is the diagonal matrix of degrees defined by $\forall i: \mathbf{D}_{i i}=\sum_{j} \mathbf{W}_{i j}$.

Thus, the normalized Laplacian of a graph is defined by [6]:

$$
\mathbf{L}_{n}=\mathbf{D}^{-1 / 2} \mathbf{L} \mathbf{D}^{-1 / 2}
$$

As $\mathbf{L}_{n}$ is a symmetric and real-valued matrix, it can be factorized using its eigenvectors as:

$$
\mathbf{L}_{n}=\mathbf{V} \boldsymbol{\Lambda} \mathbf{V}^{\top}
$$

where $\mathbf{V}$ is the orthonormal matrix whose ith column is the eigenvector of $\mathbf{L}_{n}$, $\mathbf{V}^{\top}$ is its transposed matrix, and $\boldsymbol{\Lambda}$ is the diagonal matrix whose diagonal elements are the corresponding eigenvalues, such that $\boldsymbol{\Lambda}_{i i}=\lambda_{i}$ of $\mathbf{L}_{n}$.

In the context of GSP, we define signals $\mathbf{x}$ as vectors in $\mathbb{R}^{N}$. The spectral representation of signals defined on the graph $\mathcal{G}$ can be provided using GFT [4]:

$$
\hat{\mathbf{x}}=\mathbf{V}^{\top} \mathbf{x}
$$

Columns of $\mathbf{V}$ can be interpreted as Fourier modes 4 and are relevant to describing signals with respect to typical propagation modes on the graph.

\subsection{Estimation of the structural graph (SG)}

In this paper, we consider a graph whose nodes correspond to 360 ROIs of the Glasser multimodal cortical atlas from HCP [21], and whose edges and weights are estimates of structural connectivity strength from 56 TC subjects from the HCP, using white matter tractography techniques [23]. Diffusionweighted scans were analysed using MRtrix 32 with the following operations: multi-shell multi-tissue response function estimation, constrained spherical deconvolution, tractogram generation with $10^{7}$ output streamlines. The chosen connectivity measure was the number of fibers connecting two regions divided by the region volumes (sum of connected regions). More precisely, this structural graph (SG) was obtained by averaging the 56 subjects' structural matrices.

\footnotetext{
2 http://www.mrtrix.org/
} 
No thresholds were applied to the individual subjects or average graphs. We

defined GFT using the normalized Laplacian of the averaged structural graph which is used for every patient.

In the following sections, we setup a supervised classification task that compare several statistical measures that are subsequently transformed using GFT.

\subsection{Feature extraction and Feature selection}

We extracted several features with and without the use of GFT, based on descriptive statistics of the temporal rs-fMRI signals. Namely, we compared the SD, the mean, the variance and a high-order moment, i.e. the kurtosis of rs-fMRI time series. Next, we computed the projection of the same features on the structural graph of several TC subjects in the graph Fourier domain using GFT. Moreover, for comparison purposes with the existing literature, we also extracted connectivity features via the covariance estimation of the tangent matrix [13, 24, 25], and used the lower triangular part of the resulting functional connectivity (FC) matrix. Functional connectivity (FC) provides an index of the level of co-activation of brain regions based on the time-series of rs-fMRI brain imaging data. Finally, we also used the FC matrix to compute three complex network measures known to be of interest in ASD research, namely eigenvector centrality (EC), node strength (NS) and clustering coefficient (CC) [16, 26]. These complex-graph network modeling approaches are seldom combined with supervised learning. However, they could be relevant to identify brain subsystems associated with ASD [26]. Thus, we obtained a total of twelve feature vectors, denoted by SD, SD+SG, Mean, Mean+SG, Var, Var+SG, Kurtosis, Kurtosis+SG, FC, EC, NS and CC, respectively. These feature vectors are of dimension $N$, except FC, which is the size of the lower triangular connectivity matrix, thus its size is $\frac{N(N-1)}{2}$ for each subject.

205

The main aim of this work is to present a novel modelling time series approach applied to brain imaging. In order to validate the effectiveness and robustness of the proposed method in the analysis of rs-fMRI, we tested it on a classification task, namely the diagnosis of ASD. Our analysis is based on a widely used 
pipeline for feature selection and classification [13, 25]. Precisely, in order to tween the different feature vectors to test whether the classifiers were able to predict significantly better than chance. We estimated the chance level of all trained classifiers using bootstrapping by calculating a permutation test score [27, i.e. repeating the classification procedure after randomizing the labels. This per-

\footnotetext{
${ }^{3} \mathrm{C}$ is the penalty parameter of the error term

${ }^{4} \mathrm{lbfgs}$ is the Broyden-Fletcher-Goldfarb-Shanno optimization algorithm
} 
likely predicting at chance level.

\subsection{Visualization of cross-validated selected features}

We attempted to study the stability and interpretability of the method qualitatively, by visualizing feature selection with respect to cross-validation. We calculated a vector of ratios that averages the number of times each feature is selected across folds. We then visualized these ratio back on the brain using the spatial extents of the ROI. In the case of features obtained using GFT, we applied the inverse GFT to the vector of ratios before visualization. The application of inverse GFT enables the visualization of the contribution of all regions in the atlas, as opposed to the other features, for which only selected ROI are visualized.

\section{Results and Discussion}

\subsection{Supervised Classification of $A S D$}

ABIDE datasets are heterogeneous data, which come from 17 international sites with no prior coordination, which is typical in clinical contexts. Thus, discriminating between ASD and TC individuals is a challenging task.

Figures 2, 3 and 4 reveal the averaged classification rates for the proposed method across CV-folds, as a function of the number of features when compared to different statistical measures, FC and several complex network measures using LR and SVC classifiers. The red markers, in the different approaches, indicate that the classification metrics were statistically significant $(p<0.01)$ after a permutation test using 100 permutations. The blue markers reveal the nonsignificance of the classification metrics for the different methods. Out of the different statistical metrics, several of them turned out to yield non-significant classification for all or most selected features, such as the mean of rs-fMRI. This might be justified by the fact that there are relatively short time series included in ABIDE datasets (typically 5-6 minutes per participant). However, features 
with SD+SG yielded classifiers which are highly significantly above chance level, even when selecting as few as 10 features.

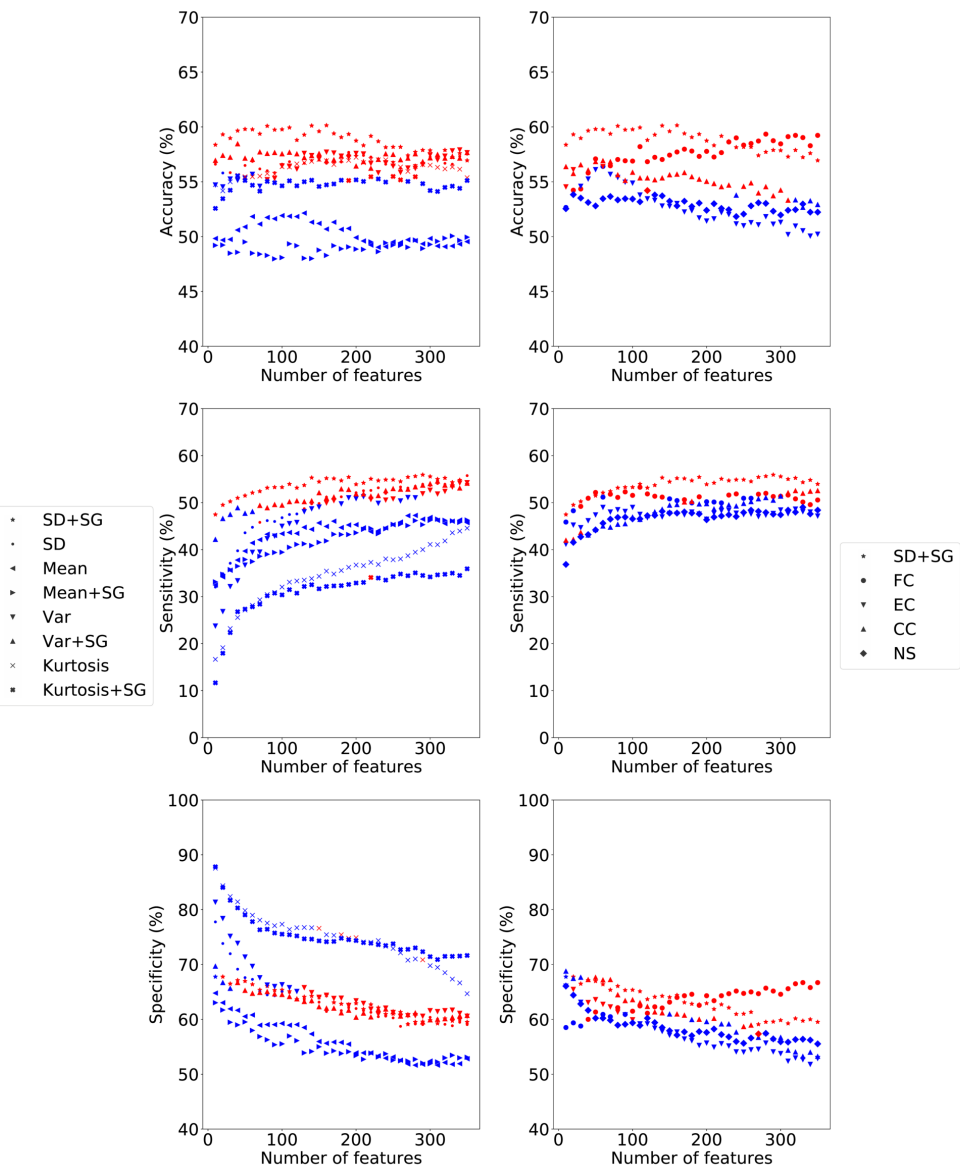

Figure 2: Left: Average accuracy, sensitivity and specificity across $20 \mathrm{CV}$-folds, as a function of number of features and statistical approaches using an LR classifier. Right: Average accuracy, sensitivity and specificity across $20 \mathrm{CV}$-folds, as a function of number of features when compared to FC and complex network measures using an LR classifier. Red markers indicate classification significantly above chance level, while blue markers indicate a lack thereof. 

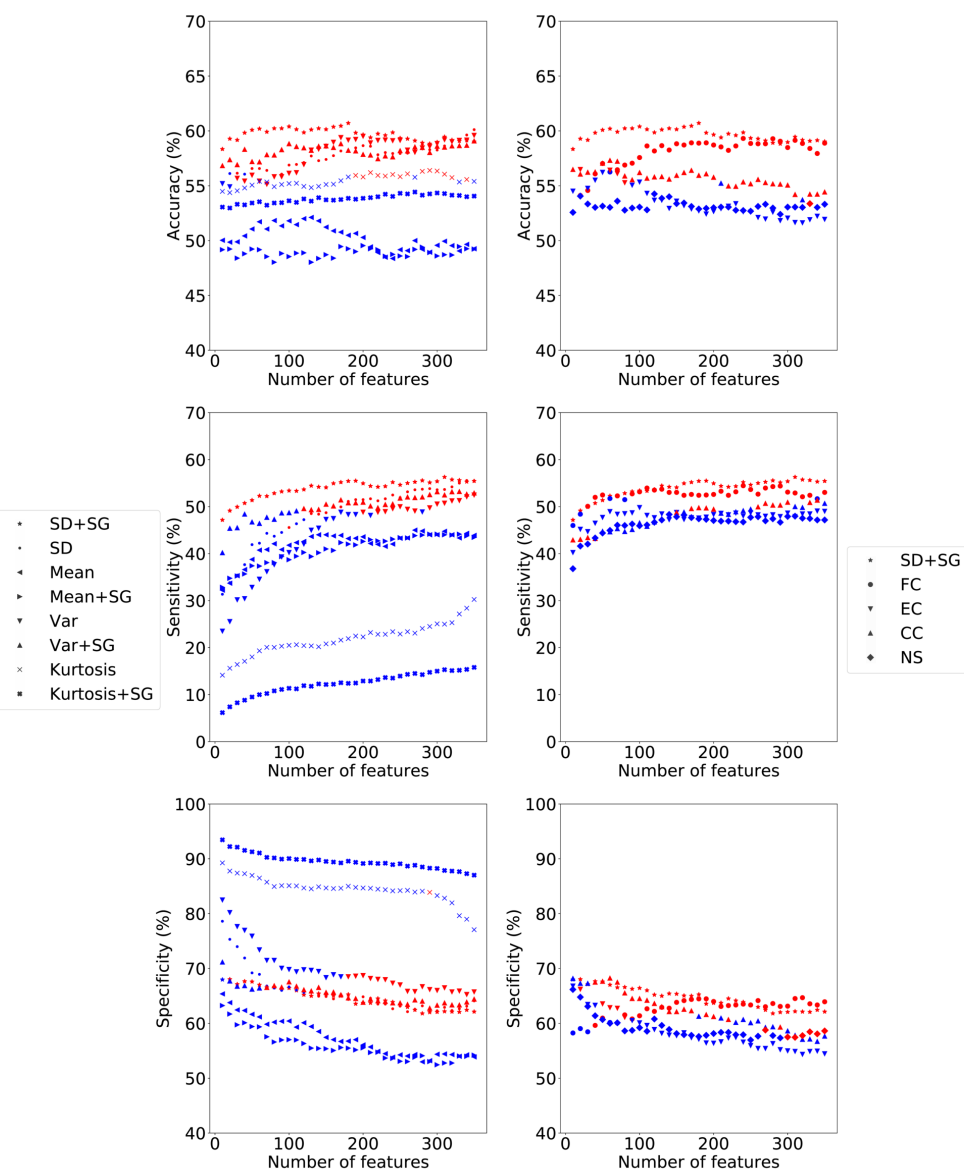

Figure 3: Left: Average accuracy, sensitivity and specificity across $20 \mathrm{CV}$-folds, as a function of number of features and statistical approaches using an SVC classifier with a linear kernel. Right: Average accuracy, sensitivity and specificity across $20 \mathrm{CV}$-folds, as a function of number of features when compared to FC and complex network measures using an SVC classifier. Red markers indicate classification significantly above chance level, while blue markers indicate a lack thereof. 

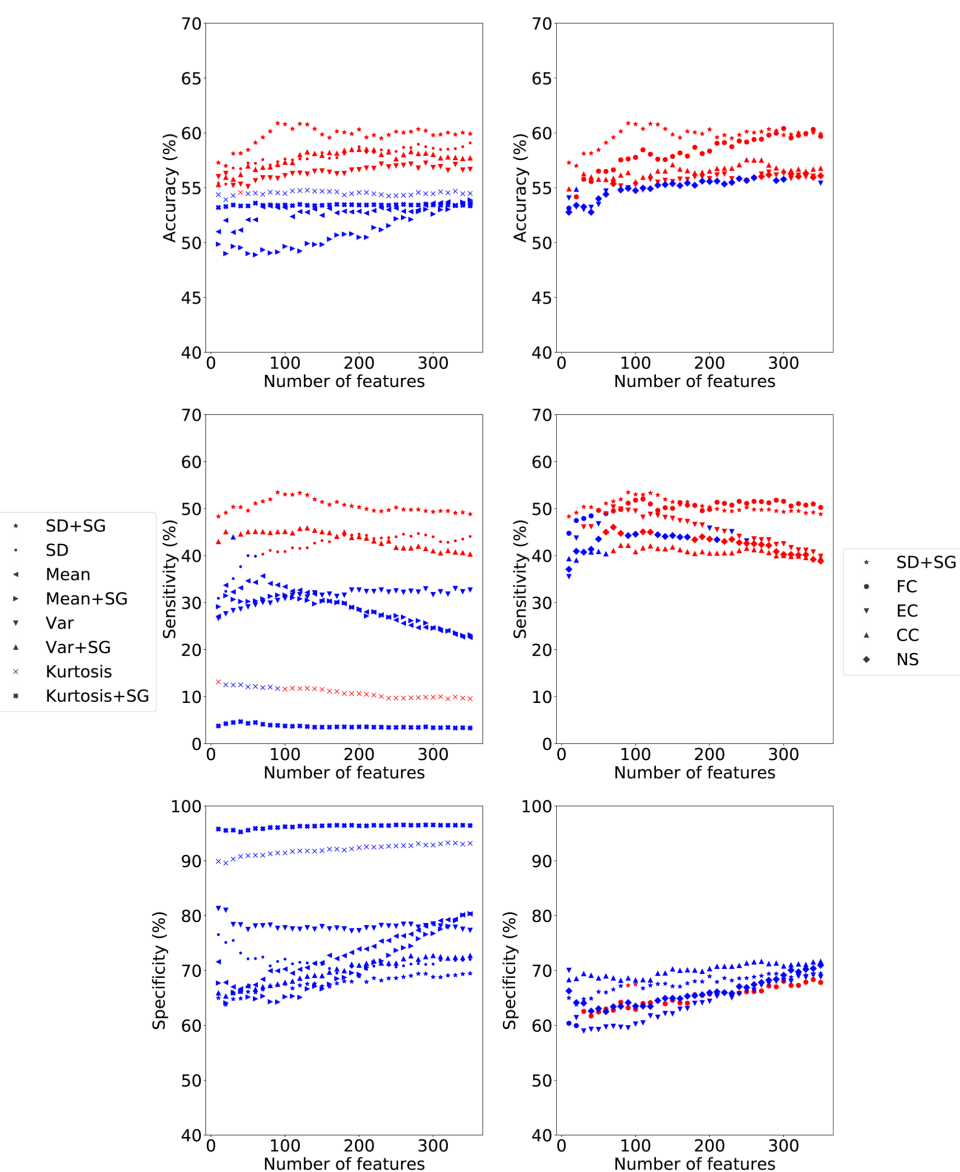

Figure 4: Left: Average accuracy, sensitivity and specificity across $20 \mathrm{CV}$-folds, as a function of number of features and statistical approaches using an SVC classifier with a RBF kernel. Right: Average accuracy, sensitivity and specificity across $20 \mathrm{CV}$-folds, as a function of number of features when compared to FC and complex network measures using an SVC classifier. Red markers indicate classification significantly above chance level, while blue markers indicate a lack thereof.

Figure 5 shows the distribution of 100 classification scores obtained by permutating labels, estimating true chance level and demonstrating that the observed accuracy is highly significantly above chance level $(p<0.01)$. In the following discussion, we will only consider approaches that lead to classifiers that are able to predict better than chance level according to this permutation scheme. 


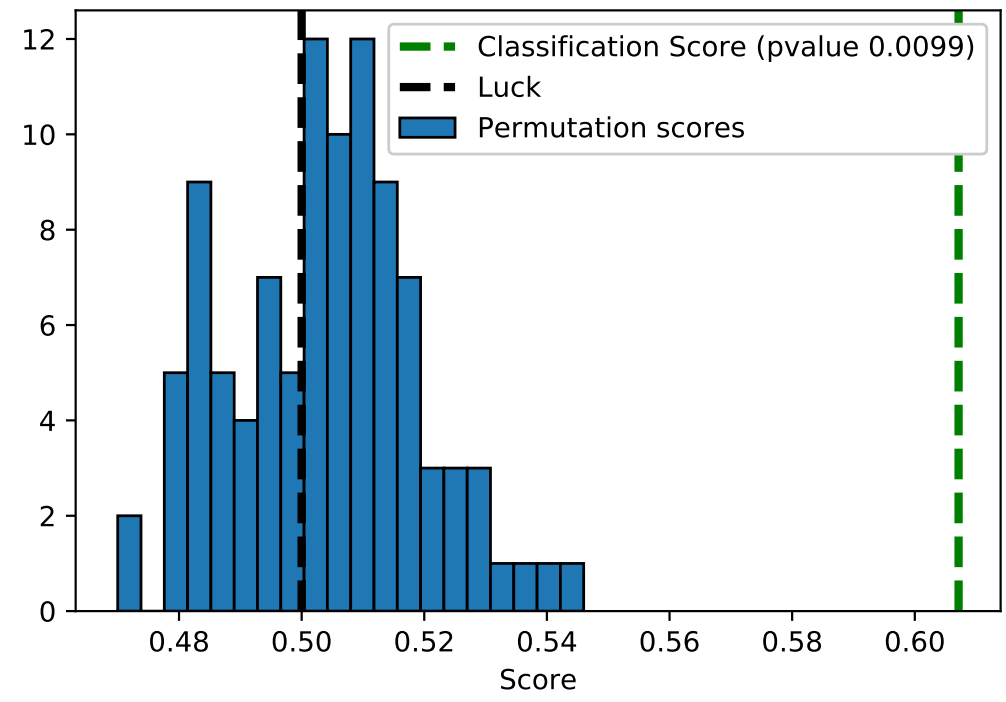

Figure 5: Permutation scores of the SD+SG approach and observed classification score (accuracy), using 100 permutations for 180 best selected feature using ANOVA.

Tables 1.2 and 3 show classification metrics for all methods that predicted better than chance level. Interestingly, feature vectors with SD+SG outperformed those obtained using other statistical metrics, functional connectivity or several complex-graph functional network modeling approaches.

Classification accuracies of $60.14 \%$ (55.92\% for sensitivity, $67.77 \%$ for specificity, permutation test $p<0.01,100$ times), $60.71 \%$ (56.30\% for sensitivity, $68.03 \%$ for specificity, permutation test $p<0.01,100$ times) and $60.89 \%$ (53.46\% for sensitivity, $69.47 \%$ for specificity, permutation test $p<0.01$, 100 times) were achieved using the SD+SG approach. When selecting the best 160, 180 and 90 features (using ANOVA) using either an LR or an SVC (linear and RBF kernels), we obtained accuracy gains of $4.4 \%, 4.31 \%$ and $6.12 \%$ respectively, when comparing the SD+SG approach with the remaining statistical methods. Moreover, when comparing the SD+SG approach with FC and several complex functional network measures, we obtained an accuracy gain up to $5.95 \%, 6,46 \%$ 


\begin{tabular}{lllr}
\hline \hline Approaches & Acc $(\%)$ & Sen $(\%)$ & Spe (\%) \\
\hline \hline SD & $60.11 \pm 0.033$ & $55.55 \pm 0.041$ & $78.61 \pm 0.047$ \\
\hline SD+SG & $\mathbf{6 0 . 7 1} \pm \mathbf{0 . 0 3 4}$ & $\mathbf{5 6 . 3 0} \pm \mathbf{0 . 0 5 1}$ & $\mathbf{6 8 . 0 3} \pm \mathbf{0 . 0 4 6}$ \\
\hline Var & $59.60 \pm 0.037$ & $52.47 \pm 0.038$ & $82.50 \pm 0.066$ \\
\hline Var+SG & $59.09 \pm 0.034$ & $53.21 \pm 0.047$ & $71.17 \pm 0.044$ \\
\hline Kurtosis & $56.4 \pm 0.021$ & $30.25 \pm 0.069$ & $89.25 \pm 0.076$ \\
\hline \hline FC & $59.31 \pm 0.040$ & $54.44 \pm 0.045$ & $64.68 \pm 0.049$ \\
\hline EC & $56.48 \pm 0.026$ & $49.81 \pm 0.052$ & $66.81 \pm 0.049$ \\
\hline NS & $54.25 \pm 0.029$ & $48.33 \pm 0.044$ & $66.17 \pm 0.064$ \\
\hline CC & $57.31 \pm 0.038$ & $51.35 \pm 0.047$ & $68.24 \pm 0.048$ \\
\hline \hline
\end{tabular}

Table 2: Maximum classification rates of the different approaches for ABIDE database using an SVC classifier with linear kernel $(\max \pm \mathrm{SD})$.

and $4,58 \%$ using the different classifiers.

\begin{tabular}{lllr}
\hline \hline Approaches & Acc (\%) & Sen (\%) & Spe (\%) \\
\hline \hline SD & $57.68 \pm 0.044$ & $55.74 \pm 0.047$ & $77.76 \pm 0.052$ \\
\hline SD+SG & $\mathbf{6 0 . 1 4} \pm \mathbf{0 . 0 3 5}$ & $\mathbf{5 5 . 9 2} \pm \mathbf{0 . 0 4 6}$ & $\mathbf{6 7 . 7 7} \pm \mathbf{0 . 0 4 2}$ \\
\hline Var & $57.91 \pm 0.040$ & $54.26 \pm 0.045$ & $81.38 \pm 0.066$ \\
\hline Var+SG & $58.46 \pm 0.032$ & $54.57 \pm 0.052$ & $69.68 \pm 0.043$ \\
\hline Kurtosis & $57.22 \pm 0.033$ & $44.56 \pm 0.064$ & $87.55 \pm 0.080$ \\
\hline Kurtosis+SG & $55.74 \pm 0.029$ & $35.92 \pm 0.062$ & $87.82 \pm 0.044$ \\
\hline \hline FC & $59.34 \pm 0.032$ & $53.27 \pm 0.055$ & $66.70 \pm 0.054$ \\
\hline EC & $56.40 \pm 0.030$ & $49.01 \pm 0.058$ & $66.06 \pm 0.048$ \\
\hline NS & $54.19 \pm 0.025$ & $49.01 \pm 0.058$ & $66.06 \pm 0.065$ \\
\hline CC & $56.91 \pm 0.040$ & $52.53 \pm 0.041$ & $68.77 \pm 0.054$ \\
\hline \hline
\end{tabular}

Table 1: Maximum classification rates of the different approaches for ABIDE database using an LR classifier ( $\max \pm \mathrm{SD})$. 


\begin{tabular}{lllr}
\hline \hline Approaches & Acc (\%) & Sen (\%) & Spe (\%) \\
\hline \hline SD & $59.09 \pm 0.034$ & $44.69 \pm 0.054$ & $76.54 \pm 0.055$ \\
\hline SD+SG & $\mathbf{6 0 . 8 9} \pm \mathbf{0 . 0 3 9}$ & $\mathbf{5 3 . 4 6} \pm \mathbf{0 . 0 5 1}$ & $\mathbf{6 9 . 4 7} \pm \mathbf{0 . 0 5 2}$ \\
\hline Var & $57.26 \pm 0.020$ & $33.02 \pm 0.088$ & $81.33 \pm 0.078$ \\
\hline Var+SG & $58.51 \pm 0.036$ & $45.86 \pm 0.066$ & $72.71 \pm 0.070$ \\
\hline Kurtosis & $54.77 \pm 0.019$ & $13.14 \pm 0.082$ & $93.29 \pm 0.043$ \\
\hline \hline FC & $60.4 \pm 0.030$ & $52.04 \pm 0.045$ & $68.35 \pm 0.052$ \\
\hline EC & $56.43 \pm 0.033$ & $50.37 \pm 0.04$ & $70.05 \pm 0.063$ \\
\hline NS & $56.31 \pm 0.032$ & $46.04 \pm 0.069$ & $70.95 \pm 0.051$ \\
\hline CC & $57.54 \pm 0.029$ & $42.16 \pm 0.064$ & $71.64 \pm 0.060$ \\
\hline \hline
\end{tabular}

Table 3: Maximum classification rates of the different approaches for ABIDE database using an SVC classifier with RBF kernel ( $\max \pm \mathrm{SD})$.

Overall, the results of the present study suggest that a first-order statistical feature [7, such as the standard deviation of rs-fMRI time series extracted using Glasser parcellation may be a discriminating feature for the classification of a mental disorder like autism. In addition, projecting this statistical metric on an average structural graph can help discriminate ASD from TC subjects, as indicated by classification metrics. Thus, these findings suggest that a multimodal neuroimaging approach may lead to greater accuracy than a single modality, such as functional connectome alone.

Furthermore, it is worth noting that the proposed approach is different from previous methods 3, 13, 16 in the classification of autism using rs-fMRI, in which the most popular approach is to exploit the whole functional connectivity matrix in the framework of functional connectome-based classification pipeline.

\subsection{Comparison with previous literature on ASD classification}

The proposed rs-fMRI analysis method was compared with several approaches for ASD diagnosis from the literature, as shown in Tables 4 and 5 . The comparison is based on three criteria, i.e. the number of subjects, the number of 
features used for the classification and the resulting accuracy values. Taking into account more than 870 subjects from the ABIDE database, the proposed approach might be comparable with the studies in [3, 13, 15, although, we did not use the optimal pipeline for feature selection and classification. These differences in feature selection/classification algorithms might account for the differences in classification accuracy between the studies. However, in the studies [11, only subjects under 20 years of age were included in their study and their model is age-dependent.

Moreover, when using the biggest subset from the full ABIDE database, such as the data from the NYU Langone Medical Center site only [18, the proposed approach outperforms current methods, such as in [3, 10, 14. Specifically, when comparing to [14, our method is able to outperform recent methods based on more fashionable techniques such as deep learning applied on hundreds of subjects. The slight difference in the accuracy value with [12], may be explained by the difference in the number of subjects for the same NYU dataset. Thus, these results were required to be validated in the whole NYU dataset using all samples.

However, it should be noted that the accuracy value of the multisite sample, i.e. the whole ABIDE database, was smaller than for the monosite sample, i.e. NYU 325 dataset. This can be justified by several factors, such as the heterogeneities in scanning protocols, called site effects, imaging sequences, acquisition parameters, and subject populations [28. Such heterogeneities will definitely limit the sensitivity for detecting abnormalities induced by ASD, resulting in a drop in accuracy value from monosite to multisite data. This commonly motivates researchers to limit the number of sites included in their analyses at the cost of sample size.

In terms of computational complexity, our method compares favorably with the existing literature, as the number of features necessary for classification is an order of magnitude smaller than methods that are based on FC, as shown in table 4 and table 5 . Precisely, the number of necessary features in our method is maximally bounded by the number of regions (for instance, 360), while methods 


\begin{tabular}{lllr}
\hline \hline Approach & $\begin{array}{l}\text { N. } \\
\text { subjects }\end{array}$ & $\begin{array}{l}\text { N. of } \\
\text { features }\end{array}$ & Accuracy (\%) \\
\hline \hline SD+SG with SVC (RBF kernel) & 871 & 90 & $\mathbf{6 0 . 8 9}$ \\
\hline Nielsen et al. 2013 [3] & 964 & 7266 & 60 \\
\hline Iidaka et al. 2015 [11] & 640 & 2728 & 90 \\
\hline Ira Ktena et al. 2017 [15] & 871 & 7260 & 62.9 \\
\hline Abraham et al. 2017 [13] & 871 & 3486 & 66.9 \\
\hline \hline
\end{tabular}

Table 4: Comparison of different approaches for classification of the ABIDE database.

based on FC use a number of features proportional to the squared number of regions.

In a nutshell, these tables reveal the reproducibility and generalizability of our proposed framework, which may work on small and even large databases, as exemplified by statistically robust gains in the classification metrics. However, from a methodological point of view, our main aim in this study is to present a novel modelling time series approach applied on rs-fMRI brain imaging, rather than the identification of biomarkers for ASD using intrinsic functional brain connectivity, as in [3, 10 16. Moreover, the analytic procedure employed in the present study represents an entirely hypothesis-free, GSP-based approach, and we provide our analysis code for replicability (see Data and Code Availability section).

\subsection{Visualizations of features}

Figure 6 depicts a visualization of 50 best features selected with ANOVA, averaged over folds. Using features from CC, SD and Var, many ROIs are consistently selected across folds as indicated by ratios close to 1 , such as the left inferior temporal cortices, right anterior and posterior cingulate cortices. Notably, the spatial reconstruction of selected features in the graph Fourier domain, estimated using inverse GFT, reveals whole brain patterns that involve all 360 ROIs. These patterns are similar for $S D+S G$ and $\operatorname{Var}+S G$, and partly 


\begin{tabular}{lllr}
\hline \hline Approach & N. of subjects & N. of features & Accuracy (\%) \\
\hline \hline SD+SG with SVC [18] & 172 & 100 & $\mathbf{7 0 . 3 6}$ \\
\hline Nielsen et al. 2013 [3] & 179 & 7266 & 65 \\
\hline \hline Dodero et al. 2015 [10] & 79 & 34,716 & 60.76 \\
\hline Wee et al. 2016 [12] & 92 & NA & 71 \\
\hline Heinsfeld et al. 2018 [14] & 175 & 19,900 & 66 \\
\hline \hline
\end{tabular}

Table 5: Comparison of different approaches for classification of a subset of the ABIDE dataset. Note that in [12], the authors only report a range regarding the number of features, corresponding to $116 \times K$, where $\mathrm{K}$ is the number of clusters considered, and is comprised between 2 and 7 . The optimal value of $\mathrm{K}$ is not specified.

overlap with the spatial locations of ratios obtained with the other features (e.g. left inferior temporal cortex), while adding other ROIs such as the left and right precentral gyri. These regions may be relevant for diagnosis of ASD, as reported in previous studies [12, 13. This visualization provides qualitative evidence that the accuracy boost obtained using GFT might be explained by the efficient combination of whole brain patterns with only a fraction of features, which results in an efficient feature selection strategy.

\subsection{Limitations and future directions}

The main limitation of this study is probably that we focus only on Glasser brain parcellations, which was generated using multimodal data, to extract regional time series and to build the structural graph. In future studies, analyses using other parcellations may be of interest and should be compared to replicate the accuracy of classification, as the choice of parcellation has previously been benchmarked as an important source of variability for classification [13.

Besides, while we used the most popular methods for classification in this database, it is likely that its findings regarding optimal pipeline decisions will carry over to other aggregate samples, as well as more homogeneous samples. Future studies may wish to validate this point, by extending this approach to larger and more homogeneous samples. Moreover, as one obvious source of het- 


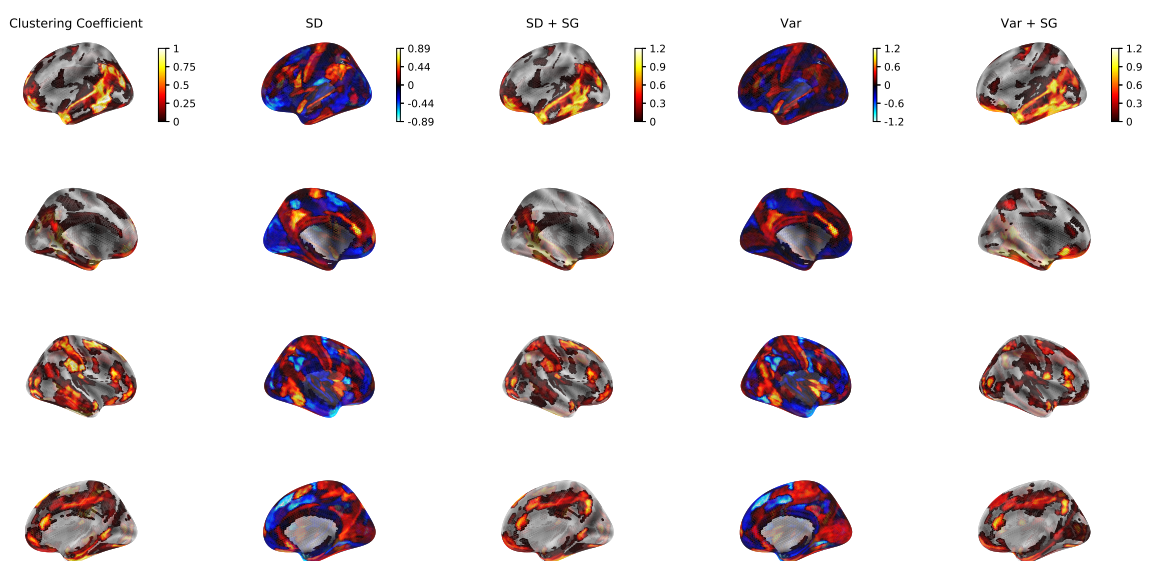

Figure 6: Spatial distributions of ratios (\%) (respectively inverse GFT of ratios for SD+SG and $\operatorname{Var}+\mathrm{SG}$ ) over 20 folds of the 50 best selected features using ANOVA. Rows correspond to different views of the cortical surface, namely left lateral, left medial, right lateral and right medial views.

erogeneity in ASD is gender [29], the results may be disproportionately skewed. Thus, future research should take into consideration this confounding variable to reduce the effects of heterogeneity for improving classification performance.

Another limitation of our work is the fact that we computed graph signals from time series using the standard deviation, but the resulting signal to noise ratio is sensitive to the length of the time series. As a consequence, a promising direction would be to address this issue by considering other statistical estimates.

Next, we limited our experiments to straightforward supervised learning methods with few parameters, namely support vector machines and logistic regression. Machine learning have recently experienced spectacular progress due to the rise of deep learning techniques, which are based on a much larger number of parameters. While other authors have proposed deep learning based approaches to classify ASD [14, we suggest that the approach proposed in the current paper could be combined with deep learning on graphs [30, as our method already extracts relevant features by exploiting the graphs using GFT. 


\section{Conclusion}

In the current study, we present a novel and efficient approach for the analysis of rs-fMRI. More specifically, this work has introduced the application of GSP

\section{Acknowledgments}

The research was partly supported by the Bretagne region, under the framework "Stratégie d'Attractivité Durable" within the project "Traitement du Signal sur Graphes". We wish to thank Maria Giulia Pretti and Dimitri Van De 
Ville for sharing their averaged structural graph. We also wish to thank Aime

Johansen for proofreading and editing the revised version of this manuscript.

[1] G. Varoquaux, B. Thirion, How machine learning is shaping cognitive neuroimaging, GigaScience 3 (1) (2014) 28.

[2] D. S. Bassett, O. Sporns, Network neuroscience, Vol. 20(3), 2017.

[3] J. A. Nielsen, B. A. Zielinski, P. T. Fletcher, A. L. Alexander, N. Lange, E. D. Bigler, J. E. Lainhart, J. S. Anderson, Multisite functional connectivity mri classification of autism: Abide results, Front Hum Neurosci 7(599).

[4] D. I. Shuman, S. K. Narang, P. Frossard, A. Ortega, P. Vandergheynst, The emerging field of signal processing on graphs: Extending high-dimensional data analysis to networks and other irregular domains, IEEE Signal Processing Magazine 30 (3) (2013) 83-98.

[5] F. R. Chung, Spectral graph theory, Vol. 92, 1997.

[6] W. Huang, T. A. W. Bolton, J. D. Medaglia, D. S. Bassett, A. Ribeiro, D. V. D. Ville, A graph signal processing perspective on functional brain imaging, Proceedings of the IEEE 106 (5) (2018) 868-885.

[7] A. Singh, M. K. Dutta, R. Jennane, E. Lespessailles, Classification of the trabecular bone structure of osteoporotic patients using machine vision, Computers in Biology and Medicine 91 (2017) 148158.

[8] S. M. Smith, D. Vidaurre, C. F. Beckmann, M. F. Glasser, M. Jenkinson, K. L. Miller, T. E. Nichols, E. C. Robinson, G. Salimi-Khorshidi, M. W. Woolrich, et al., Functional connectomics from resting-state fmri, Trends in cognitive sciences 17 (12) (2013) 666-682.

[9] M. D. Fox, M. Greicius, Clinical applications of resting state functional connectivity, Frontiers in systems neuroscience 4 (2010) 19.

[10] L. Dodero, H. Q. Minh, M. San Biagio, V. Murino, D. Sona, Kernel-based classification for brain connectivity graphs on the riemannian manifold of 
positive definite matrices, in: 2015 IEEE 12th International Symposium on Biomedical Imaging (ISBI), IEEE, 2015, pp. 42-45.

[11] T. Iidaka, Resting state functional magnetic resonance imaging and neural network classified autism and control, Cortex 63 (2015) 55-67.

[12] C.-Y. Wee, P.-T. Yap, D. Shen, Diagnosis of autism spectrum disorders using temporally distinct resting-state functional connectivity networks, CNS neuroscience \& therapeutics 22 (3) (2016) 212-219.

[13] A. Abraham, M. P. Milham, A. D. Martino, R. C. Craddock, D. Samaras, B. Thirion, G. Varoquaux, Deriving reproducible biomarkers from multisite resting-state data: An autism-based example, NeuroImage 147 (2017) $736-745$.

[14] A. S. Heinsfeld, A. R. Franco, R. C. Craddock, A. Buchweitz, F. Meneguzzi, Identification of autism spectrum disorder using deep learning and the abide dataset, NeuroImage. Clinical 17 (2018) 16-23.

[15] S. I. Ktena, S. Parisot, E. Ferrante, M. Rajchl, M. C. H. Lee, B. Glocker, D. Rueckert, Distance metric learning using graph convolutional networks: Application to functional brain networks, in: International Conference on Medical Image Computing and Computer-Assisted Intervention, Vol. abs/1703.02161, 2017. arXiv:1703.02161.

[16] A. Kazeminejad, R. C. Sotero, Topological properties of resting-state fmri functional networks improve machine learning-based autism classification, Frontiers in Neuroscience 12 (2019) 1018.

[17] M. F. Glasser, T. S. Coalson, E. C. Robinson, C. D. Hacker, J. Harwell, E. Yacoub, K. Ugurbil, J. Andersson, C. F. Beckmann, M. Jenkinson, et al., A multi-modal parcellation of human cerebral cortex, Nature 536 (7615) (2016) 171.

[18] A. Brahim, M. Hajjam El Hassani, N. Farrugia, Classification of autism spectrum disorder through the graph fourier transform of fmri temporal 
signals projected on structural connectome, in: M. Vento, G. Percannella,

S. Colantonio, D. Giorgi, B. J. Matuszewski, H. Kerdegari, M. Razaak (Eds.), Computer Analysis of Images and Patterns, Springer International Publishing, Cham, 2019, pp. 45-55.

[19] A. Di Martino, C. G. Yan, Q. Li, E. Denio, F. X. Castellanos, K. Alaerts, J. S. Anderson, M. Assaf, S. Y. Bookheimer, M. Dapretto, B. Deen, S. Delmonte, I. Dinstein, B. Ertl-Wagner, D. A. Fair, L. Gallagher, D. P. Kennedy, C. L. Keown, C. Keysers, J. E. Lainhart, C. Lord, B. Luna, V. Menon, N. J. Minshew, C. S. Monk, S. Mueller, R. A. Mller, M. B. Nebel, J. T. Nigg, K. O'Hearn, K. A. Pelphrey, S. J. Peltier, J. D. Rudie, S. Sunaert, M. Thioux, J. M. Tyszka, L. Q. Uddin, J. S. Verhoeven, N. Wenderoth, J. L. Wiggins, S. H. Mostofsky, M. P. Milham, The autism brain imaging data exchange: towards a large-scale evaluation of the intrinsic brain architecture in autism., Mol Psychiatry 19(6) (2014) 659-67.

[20] C. Craddock, S. Sikka, B. Cheung, R. Khanuja, S. S. Ghosh, C. Yan, Q. Li, D. Lurie, J. Vogelstein, R. Burns, S. Colcombe, M. Mennes, C. Kelly, A. Di Martino, F. X. Castellanos, M. Milham, Towards automated analysis of connectomes: The configurable pipeline for the analysis of connectomes (c-pac), Frontiers in Neuroinformatics (42).

[21] M. F. Glasser, T. S. Coalson, E. C. Robinson, C. D. Hacker, J. Harwell, E. Yacoub, K. Ugurbil, J. Andersson, C. F. Beckmann, S. S. M. Jenkinson, M., D. C. Van Essen, A multi-modal parcellation of human cerebral cortex, Nature vol. 536,7615 (2016): 171-178. 536 (7615) (2016) 171-178.

[22] W. Huang, L. Goldsberry, N. F. Wymbs, S. T. Grafton, D. S. Bassett, A. Ribeiro, Graph frequency analysis of brain signals, IEEE Journal of Selected Topics in Signal Processing 10 (7) (2016) 1189-1203.

[23] M. G. Preti, D. V. D. Ville, Decoupling of brain function from structure reveals regional behavioral specialization in humans, Nature Communications 10 (2019) 4747. arXiv:1905.07813v2. 
[24] G. Varoquaux, F. Baronnet, A. Kleinschmidt, P. Fillard, B. Thirion, Detection of brain functional-connectivity difference in post-stroke patients using group-level covariance modeling, in: Medical Image Computing and Computer-Assisted Intervention, 2010.

[25] K. Dadi, M. Rahim, A. Abraham, D. Chyzhyk, M. Milham, B. Thirion, G. Varoquaux, Benchmarking functional connectome-based predictive models for resting-state fmri, NeuroImage 192 (2019) 115 - 134.

[26] J. R. Sato, C. V. M., S. de Siqueira Santos, K. Brauer Massirer, A. Fujita, Complex network measures in autism spectrum disorders, IEEE/ACM Trans Comput Biol Bioinform 15(2) (2018) 581-587.

[27] P. Golland, B. Fischl, Permutation tests for classification: towards statistical significance in image-based studies, in: Information processing in medical imaging, 2003, pp. 330-341.

[28] C. Dansereau, Y. Benhajali, C. Risterucci, E. M. Pich, P. Orban, D. Arnold, P. Bellec, Statistical power and prediction accuracy in multisite resting-state fmri connectivity, NeuroImage 149 (2017) 220 - 232. doi:https://doi.org/10.1016/j.neuroimage.2017.01.072. URL http://www.sciencedirect.com/science/article/pii/ S1053811917300939

[29] M. C. Lai, M. V. Lombardo, J. Suckling, A. N. Ruigrok, B. Chakrabarti, C. Ecker, S. Deoni, M. Craig, D. Murphy, E. Bullmore, M. A. Consortium, S. Baron-Cohen, Biological sex affects the neurobiology of autism, Brain : a journal of neurology 136(9) (2013) 2799-2815.

[30] Z. Wu, S. Pan, F. Chen, G. Long, C. Zhang, P. S. Yu, A comprehensive survey on graph neural networks, arXiv preprint arXiv:1901.00596.

[31] F. Pedregosa, G. Varoquaux, A. Gramfort, V. Michel, B. Thirion, O. Grisel, M. Blondel, P. Prettenhofer, R. Weiss, V. Dubourg, J. Vanderplas, A. Pas$530 \quad \square$ sos, D. Cournapeau, M. Brucher, M. Perrot, E. Duchesnay, Scikit-learn: 
Machine learning in python, J. Mach. Learn. Res. 12 (2011) 2825-2830.

URL http://dl . acm.org/citation. cfm?id=1953048.2078195

[32] A. Abraham, F. Pedregosa, M. Eickenberg, P. Gervais, A. Mueller, J. Kossaifi, A. Gramfort, B. Thirion, G. Varoquaux, Machine learning for neuroimaging with scikit-learn, Frontiers in Neuroinformatics 8 (2014) 14. doi:10.3389/fninf.2014.00014. 